\title{
Convergência de Renda Per Capita entre os Municípios Nordestinos: uma Análise Robusta*
}

\section{Per Capita Income Convergence among Northeastern Municipalities: a Robust Analysis}

\author{
Cleiton Roberto da Fonseca Silva** \\ Erik Alencar de Figueiredo***
}

\begin{abstract}
Resumo: Neste artigo, foram comparados os vários métodos de inferência referentes à hipótese de convergência de renda per capita usando como base os dados dos municípios nordestinos para o período de 1970 a 1996. Demonstrou-se que, embora a análise quantílica linear resolva a maior parte dos problemas inerentes às regressões via mínimos quadrados ordinários, não consegue captar o processo de divergência entre as curvas quantílicas que caracteriza a formação de clubes de convergência. Nesse sentido, esse estudo utiliza as regressões quantilicas não paramétricas, conforme proposto por Laurini (2007). Concluiu-se que o período em análise é caracterizado pela formação de clubes de convergência entre os municípios nordestinos.
\end{abstract}

Palavras-chave: Municípios nordestinos. Convergência de renda. Regressão quantílica. Splines quantílicos.

Abstract: In this paper it was evaluated several methods of inference about income
convergence hypothesis exploring the database of Brazilian northeast municipali-
ties through the 1970-1996 period. The results show that linear quantile regression
does not capture the divergences between quantilic curves that characterize the
formation of convergence clubs, although its use can solve many difficulties into
least squares fitting. In this direction, this study applies nonparametric quantile re-
gression known as quantile smoothing splines. We can conclude to that period that
northeast municipalities are better characterized by clubs convergence formation.

Keywords: Brazilian northeast municipalities. Income convergence. Quantile regression. Quantile smoothing splines.

JEL Classification: $\mathrm{O} 41 ; \mathrm{C} 31$.

\footnotetext{
* O primeiro autor agradece o suporte financeiro da Capes/Reuni. O segundo autor gostaria de agradecer o suporte financeiro do CNPq, por meio do Projeto 474227/2007. Estamos em débito com o professor Márcio P. Laurini; contudo, erros e omissões são de nossa inteira responsabilidade.

** Aluno do curso de Mestrado em Economia da UFPB. Email: cleiton.roberto@yahoo.com.br

*** Professor do Programa de Pós-graduação em Economia da UFPB. Email: eafigueiredo@ gmail.com
} 


\section{Introdução}

As investigações empíricas relacionadas à convergência de renda já ocuparam um grande espaço na literatura especializada. ${ }^{1}$ Em resumo, os estudos costumam seguir o modelo sugerido por Barro (1991), ou seja:

$$
\gamma_{i}=\beta \log \left(y_{i, 0}\right)+\alpha X_{i}+\phi Z_{i}+\varepsilon_{i}
$$

onde $\gamma_{i}$ é a taxa de crescimento do produto per capita para a economia $i, y_{i, 0}$ é a renda per capita da economia i no ano inicial, $X_{i}$ sumariza as variáveis explicativas para a taxa de crescimento e $Z_{i}$ é o conjunto de variáveis que afetam o crescimento, mas não estão diretamente relacionadas com o modelo teórico.

Normalmente, a coleção de variáveis explicativas é apontada por estruturas teóricas a la Solow (1956). ${ }^{2}$ Nessas estruturas, a observação de um parâmetro $\beta<0$ indica que quanto maior a dotação inicial $\left(y_{i, 0}\right)$ menor a taxa de convergência para o estado estacionário. Ou seja, as economias pobres tendem a apresentar uma maior taxa de convergência em relação às economias ricas.

O fato é que, independente das unidades econômicas envolvidas (países, estados ou municípios), grande parte da literatura costuma inferir os parâmetros de (1) a partir de regressões baseadas em Mínimos Quadrados Ordinários (MQO). Contudo, esta estratégia está sujeita a, pelo menos, duas críticas. Primeiro, um $\beta<0$ pode não indicar convergência e sim uma mera regressão à média, situação conhecida como falácia de Galton. ${ }^{3}$ Segundo, as presenças de outliers e heterocedasticidade podem viesar os coeficientes da regressão. No mais, a equação (1) assume um parâmetro único. Ou seja, considera que todas as economias possuem a mesma taxa de convergência, sumarizadas por $\beta$.

No entanto, todos estes problemas podem ser contornados a partir da consideração de modelos de regressão quantílica. Esta afirmação é assegurada por Koenker (2000), que enfatiza a robustez deste ferramental frente à falácia de Galton, outliers e heterocedasticidade. No mais, a própria estrutura quantílica é capaz de identificar possíveis diferenciações entre os parâmetros $\beta$ 's, contornando o problema da taxa de convergência única entre as unidades investigadas. ${ }^{4}$ Adicionalmente, o modelo de regressões quantílicas permite testar a hipótese de Clubes de Convergência, conforme demonstrado em Andrade et al. (2005).

\footnotetext{
Para uma síntese, ver Sala-i-Martin (1996).

Um conjunto de variáveis explicativas pode ser encontrado em Sala-i-Martin (1996).

Para detalhes, ver Friedman (1992) e Quah (1993).

O pioneirismo da aplicação deste método na análise da beta convergência entre as nações pode ser atribuído a Melo e Novo (2002). No Brasil, destaca-se Andrade et al. (2002).
} 
A ideia de Clubes de Convergência foi introduzida por Quah (1997). Em suma, postula-se que as economias tendem a formar blocos, convergindo para estados estacionários diferenciados. Neste caso, a estrutura (1) é novamente comprometida. A alternativa empírica adotada por Quah (1997) baseou-se nas cadeias de Markov de primeira ordem. Sendo assim, considerou-se que a distribuição das rendas per capita é governada por um processo estocástico representado por uma matriz de transição que, sob certas circunstâncias, convergirá para um equilíbrio independente do ponto inicial.

De fato, a hipótese dos Clubes de Convergência tem sido corroborada na literatura empírica. No caso específico do Brasil, têm-se as evidências contidas em Laurini (2007). Já Pôrto Junior e Ribeiro (2003) adotam o modelo de cadeias de Markov, aplicando-o aos municípios nordestinos e, também, detectam a presença de Clubes de Convergência.

Contudo, não obstante à importância dos modelos paramétricos de regressões quantílicas e de Cadeias de Markov, estas estruturas não estão isentas de críticas. Recentemente, Laurini (2007) discutiu uma importante limitação do modelo paramétrico de regressões quantílicas, qual seja: seu processo de estimação assume uma relação linear entre cada quantil da taxa de crescimento do produto e a renda inicial. Caso esta especificação esteja incorreta, os estimadores para os parâmetros serão viciados.

Do outro lado, os modelos baseados em Cadeias de Markov, embora captem importantes não linearidades, são baseados em discretizações ad hoc dos dados. Ou seja, ao estabelecer os limiares da matriz de transição, o pesquisador interfere na estrutura dos dados sem obedecer a nenhuma regra pré-estabelecida. ${ }^{5}$

Com vistas a contornar estes dois problemas, este estudo adotará a modelagem quantílica não linear conhecida como constrained B-spline smoothing (COBS). Este instrumental não paramétrico é derivado do spline quantílico proposto por Koenker, NG e Portnoy (1994). Sendo assim, adota-se o princípio de que "os dados falem por si", sem nenhuma imposição a priori sobre sua estrutura. E mais, a estimação das curvas servirá como informação sobre o comportamento da regressão em cada estrato, tomados de forma contínua, ou seja, sem discretizações ad hoc.

Isto posto, o objetivo deste estudo é testar a hipótese da convergência de renda entre os municípios nordestinos. Para tanto, serão conjugados vários métodos de estimação robustos divididos em duas partes: primeiro, implementa-se uma análise tradicional comparando os métodos de MQO e regressões quantílicas; em seguida, adota-se a estratégia sugerida por Laurini (2007), comparando-se as inferências quantílicas paramétrica e não paramétrica.

5 Morillo (1999) apresenta uma boa discussão relacionada a este tópico. 


\section{Dados e Análise Preliminar}

Para o estudo, tomam-se como base os dados fornecidos pelo Instituto de Pesquisa Econômica Aplicada (IPEA) para os anos de 1970, 1980 e 1996. Durante esse período, municípios emanciparam, outros foram extintos e alguns possuem dados insuficientes de forma que um arranjo fez-se necessário. Inicialmente, a unidade federativa que desmembrou de apenas um município teve seus dados agregados ao município de origem. Aqueles que desmembraram de dois ou mais mereceram cuidado especial: os que emanciparam nos anos da década de 1990 e, portanto, ainda possuíam os dados agregados ao município original, foram mantidos. No entanto, aqueles cujos dados já estavam desagregados foram eliminados devido à impossibilidade de agregação. O mesmo procedimento fez-se necessário para os casos relacionados aos municípios extintos ou cujos dados eram insuficientes. Informações detalhadas podem ser encontradas no Quadro 1, no Apêndice.

O primeiro passo será estimar as densidades para as rendas per capita nos três anos considerados. Para tanto, utilizar-se-á o método não paramétrico kernel smoothing. Este método permite a visualização de "fotografias" da distribuição dos rendimentos em períodos distintos, possibilitando também a implementação de testes que indiquem possíveis mudanças na estrutura dos dados e sua significância estatística.

Na sua forma mais simples, os estimadores kernel são "alisamentos" de histogramas, onde os dados, situados em uma determinada "vizinhança" do ponto $x$, são utilizados para inferir sobre a distribuição da variável de interesse. Sua propriedade não paramétrica torna-se imprescindível quando não se tem o conhecimento do processo gerador dos dados. Neste caso, adota-se o princípio de que "os dados falem por si", sem a necessidade de suposições a priori sobre sua distribuição. O estimador kernel de uma função densidade assume a seguinte forma:

$$
f_{h}=\frac{1}{N} \sum_{t=1}^{N} K_{h}\left(x-x_{t}\right)
$$

onde $K_{h}(u)=h^{-1} K(u / h)$, sendo $K$ uma função kernel simétrica que satisfaz $\int K(x) d x=1$; $N$ é o tamanho da amostra e $h$ corresponde ao parâmetro de alisamento ou janela (bandwidth). ${ }^{6}$ Optou-se pela escolha do K ótimo

6 Ziegelmann (2003) demonstra que a escolha de $h$ envolve um trade off entre variância e viés para o $f_{h}$. Em suma, um grande $h$ produz uma pequena variância e um grande viés para $f_{h}$, ocorrendo o inverso quando se tem um pequeno $h$. Logo, a determinação de $h$ toma lugar de destaque na estimação das densidades. Vários métodos de seleção da janela são sugeridos na literatura. Este estudo opta por um método plug-in simples. Para detalhes, ver Härdle (1990). 
dado por: ${ }^{7}$

$$
K^{*}(x)=(3 / 4)\left(1-x^{2}\right) I(|x|<1)
$$

A expressão acima corresponde ao kernel Epanechnikov, onde $x$ é o argumento da função kernel e I é a variável indicadora, que assume valor um quando o argumento é verdadeiro e valor zero caso esse seja falso.

As estimações para as densidades podem ser visualizadas na Figura 1. A primeira característica marcante é o deslocamento das densidades para a direita em relação ao ano inicial. Contudo, a densidade do ano intermediário, 1980, apresenta uma maior renda média além de uma maior concentração de municípios no lado direito da densidade. Neste sentido, a transição da renda entre os anos de 1980 e 1996 parece ter sido desfavorável aos municípios com renda imediatamente superiores à média. Em suma, a inspeção visual indica uma mudança expressiva na distribuição da renda per capita entre os municípios nordestinos no período em questão.

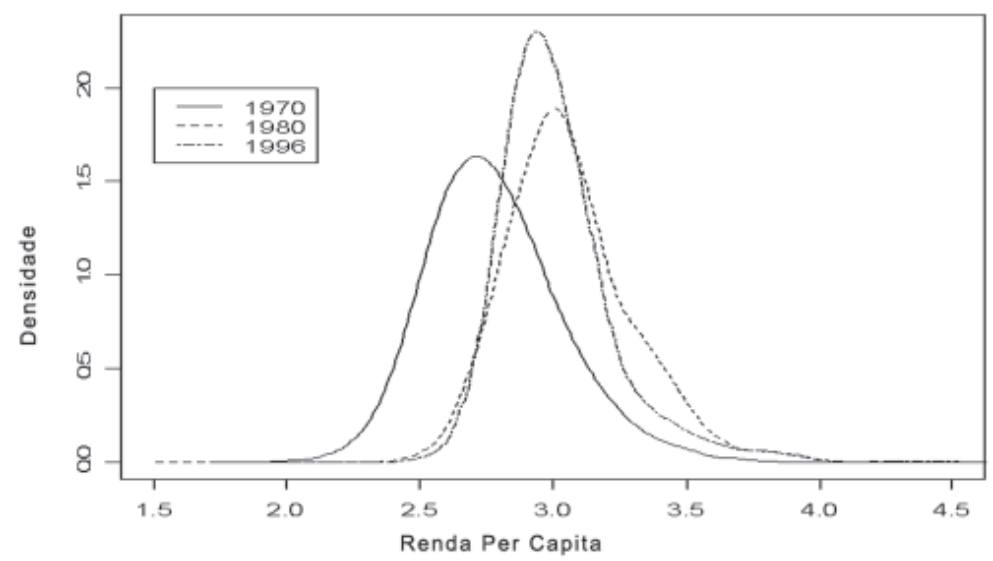

Figura 1 - Densidades Não Paramétricas para as Rendas per capita: 1970, 1980 e 1996.

Fonte: Elaborada pelos autores.

Outras informações relevantes podem ser obtidas na Tabela 1. Nela, estão dispostos os resultados relacionados à concentração e à polarização de renda. A medida de concentração é calculada a partir do conhecido índice de Gini. Já os resultados de polarização são obtidos pelo método robusto proposto por Duclos, Esteban e Ray (2004). A estimativa para o índice de polarização é dada por:

$7 \quad$ O K ótimo é obtido a partir da minimização do erro quadrático médio assintótico integrado. Para maiores detalhes ver Ziegelmann (2003). 


$$
\begin{gathered}
P_{\tau}(F)=N^{-1} \sum_{i=1}^{n} f\left(y_{i}\right)^{\tau} \hat{a}\left(y_{i}\right), \text { onde } \\
\hat{a}\left(y_{i}\right)=\mu+y_{i}\left[N^{-1}(2 i-1)-1\right]-N^{-1}\left(2 \sum_{j=1}^{i-1} y_{j}+y_{i}\right)
\end{gathered}
$$

Nesse caso, $y$ corresponde à renda, $\mu$ é a média amostral, $F$ a função de distribuição empírica, $f\left(y_{i}\right)^{\tau}$ é inferido não paramétricamente utilizando um estimador kernel simétrico, $\tau \in(0.25,1)$ representa o parâmetro de threshold. Sendo assim, define-se $f(y) \equiv N^{-1} \sum_{i=1}^{N} K_{h}\left(y-y_{i}\right), \operatorname{com}_{h}(z) \equiv h^{-1} K$ $(z / h)$ e $h$ correspondendo ao parâmetro de suavização. ${ }^{8} \mathrm{O}$ parâmetro ótimo $h$ " será obtido de modo a minimizar o erro quadrático médio do estimador, ou seja:

$$
h^{*} \cong 4.7 N^{-0.5} \sigma \tau^{0.1}
$$

Onde, $\sigma$ corresponde à variância da renda.

Tabela 1 - Índices de Concentração e Polarização de Renda per capita

\begin{tabular}{c|c|c|c}
\hline Índices & $\mathbf{1 9 7 0}$ & $\mathbf{1 9 8 0}$ & $\mathbf{1 9 9 6}$ \\
\hline Gini & 0.5949 & 0.3856 & 0.3423 \\
\hline Polarização* $^{*}$ & 0.0850 & 0.1891 & 0.2082 \\
\hline
\end{tabular}

* parâmetro de threshold $\tau=1$.

Fonte: Elaborada pelos autores a partir dos dados da pesquisa.

Os resultados da Tabela 1 apontam para uma redução expressiva do índice de concentração de renda. Isto é perfeitamente compatível com a convergência de renda, já que, se os municípios estão caminhando para um estado estacionário único, é de se esperar que a desigualdade entre eles diminua ao longo do tempo. Contudo, a observação do índice de polarização sugere que estas rendas estão convergindo para polos diferentes. Nota-se que há uma elevação considerável neste indicador, o que é condizente com a formação de Clubes. As seções seguintes tentarão estabelecer evidências mais confiáveis para esse comportamento.

\section{Resultados Lineares}

Nesta seção, serão estabelecidos os resultados lineares do paper. O objetivo central dessa estratégia é confrontar as estimações via MQO

8 Vale salientar que o processo de estimação considera uma função kernel gaussiana (contínua e diferenciável), dada por: $K(u)=(2 \pi)^{-0.5} \exp ^{-0.5 u^{2}}$. 
e regressões quantílicas. Para tanto, considerar-se-á um procedimento empírico básico dos estudos de beta convergência, qual seja, obter estimativas para a equação:

$$
\left(\frac{1}{N}\right) \log \left(\frac{y_{i N}}{y_{i 0}}\right)=\alpha+\beta \log \left(y_{i 0}\right)+u_{i 0, N}
$$

onde $\alpha$ e $\beta$ são constantes, $y_{i 0}$ e $y_{i N}$ são as rendas per capita dos municípios i's nos anos inicial e final, respectivamente e $u_{i 0, N}$ representa os erros médios entre o período 0 e $N$. Note que se está considerando a taxa de crescimento média da renda entre o tempo 0 eN. Sabe-se que valores de $\beta<0$ denotam convergência absoluta.

As estimativas de MQO conduziram aos seguintes resultados:

$$
\left(\frac{1}{N}\right) \log \left(\frac{y_{i N}}{y_{i 0}}\right)=0.0724-0.0228 \log \left(y_{i 0}\right)
$$

com os dois parâmetros significativos a 1\%. Esta estimativa indica que há convergência absoluta entre os municípios nordestinos. De fato, ela é bem próxima à encontrada por Andrade et al. (2002). Convém ressaltar que os testes usuais indicam as presenças de autocorrelação e heterocedasticidade. Contudo, como já explicitado anteriormente, este não é o único problema das estimativas via MQO. Por conta disso, opta-se por estimar (7) a partir do modelo quantílico linear.

A estrutura teórica de regressões quantílicas foi proposta inicialmente por Koenker e Bassett (1978). Em resumo, considera-se a seguinte função objetivo: ${ }^{9}$

$$
\min _{\beta \in \in^{p}} \sum_{i=1}^{N} \rho_{\tau}\left(y_{i}-x_{i} \beta_{\tau}\right)
$$

onde $\rho_{\tau}$ é a função perda condicionada ao quantil $\tau \in(0,1)$. Define-se a função perda por $\rho_{\tau}(u)=u(\tau-I(u<0))$, com I correspondendo à variável indicadora e $u$ representando a diferença entre o valor observado e estimado de cada observação. A otimização de (9) fornecerá os parâmetros $\beta_{\tau}$. Neste sentido, o problema (7) pode ser escrito em termos dos quantis condicionais, isto é:

$$
\min _{\beta \in \in^{p}} \sum_{i=1}^{N} \rho_{\tau}\left(y_{i}-\beta_{\tau} \log \left(y_{i 0}\right)\right)
$$

9 Para uma visão geral sobre regressões quantilicas, incluindo os fundamentos, a teoria assintótica, os modelos não lineares, os aspectos computacionais, entre outros, ver Koenker (2005). 
A análise da hipótese da convergência de renda à luz das regressões quantîlicas não é uma novidade. Os estudos de Melo e Novo (2002), Melo e Perrelli (2003) e Andrade et al. (2002) servem como uma boa referência para o tema. Em suma, as estimações de (10) fornecerão o valor de $\beta_{\tau}$ para cada quantil da distribuição.

Os resultados para as regressões quantílicas estão sumarizados na Tabela 2. Optou-se por apresentar os coeficientes relativos aos quantis $0.05,0.10,0.25,0.50,0.75,0.90$ e 0.95 . A primeira constatação é que os parâmetros da regressão quantílica diferem da estimativa de MQO. E mais, há uma diferenciação na inclinação entre os quantis, onde se observa que quanto menor o quantil, maior o parâmetro em valor absoluto. Isto é, quanto menor a renda inicial, maior a velocidade de convergência.

Tabela 2 - Resultados das Regressões Quantílicas para Convergência Absoluta

\begin{tabular}{c|c|c}
\hline Quantis & Beta & Erro Padrão \\
\hline 5 & $-0.03195^{*}$ & 0.00217 \\
\hline 10 & $-0.03008^{*}$ & 0.00110 \\
\hline 25 & $-0.02893^{*}$ & 0.00094 \\
\hline 50 & $-0.02482^{*}$ & 0.00067 \\
\hline 75 & $-0.02136^{*}$ & 0.00105 \\
\hline 90 & $-0.01653^{*}$ & 0.00273 \\
\hline 95 & $-0.01444^{*}$ & 0.00228 \\
\hline
\end{tabular}

Nota: *Resultados significativos a 1\%. ·Erros padrões calculados via bootstrap. Fonte: Elaborada pelos autores.

Contudo, esta diferença entre os parâmetros de inclinação é estatisticamente significativa? Para responder a esta questão adota-se uma extensão do teste de Wald proposta por Koenker e Bassett (1982). A estatística F igual 13.07 com um p-value próximo a zero indica que a diferença entre os quantis especificados na Tabela 2 é estatisticamente significativa.

Por fim, a representação gráfica das regressões da Tabela 2 pode ser observada na Figura 2. Sendo assim, pode-se inferir que a hipótese da convergência absoluta de renda é compatível com os dados nordestinos no período de 1970 a 1996. E mais, esta estimativa mostra-se robusta frente à falácia de Galton e à presença de heterocedasticidade.

Entretanto, não há nada de novo nesses resultados, dado que já foram apontados por estudos anteriores, em especial Andrade et al. (2002). Desta forma, seguindo Laurini (2007), questiona-se: a existência de não linearidades pode viesar esta conclusão? A seção seguinte tratará deste ponto. 


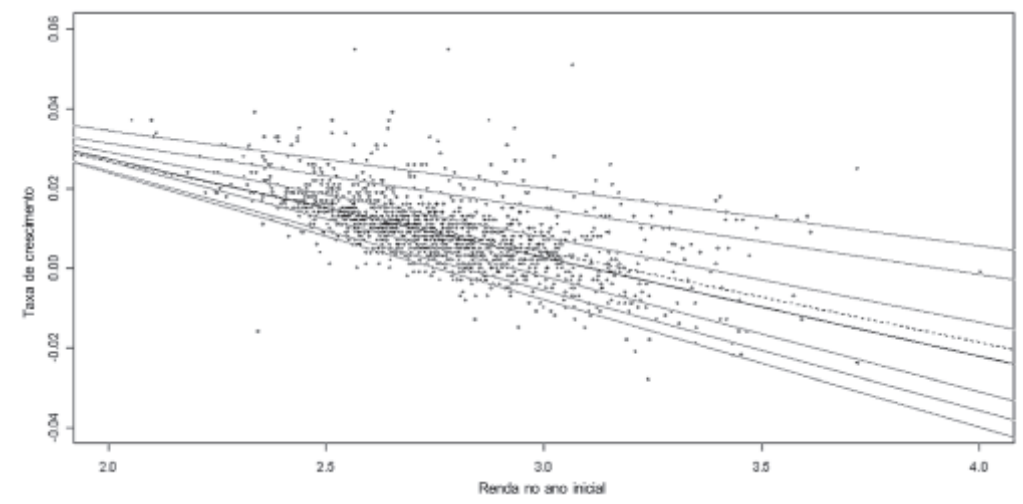

Figura 2 - Regressão Quantílica

Fonte: Elaborada pelos autores.

\section{Resultados Não Lineares}

A pergunta central desta seção é: os dados dos PIBs per capita municipais seguem um comportamento linear? Como já ressaltado, caso a resposta seja negativa as estimativas da Seção 3 podem apresentar importantes vieses.

Um forte indício de não linearidade é apontado por Porto Junior e Ribeiro (2003). Estes autores detectam, a partir de um modelo de Cadeias de Markov, a presença de um comportamento não linear na distribuição das rendas per capita dos municípios nordestinos no período compreendido entre 1970 e 1996. E mais, a matriz de transição resultante aponta para a formação de dois grupos de municípios: um pobre, com rendas médias abaixo da renda média regional, e um rico, com rendas médias superiores à renda média regional. Logo, surge a hipótese dos Clubes de Convergência.

Contudo, as probabilidades de transição inferidas via Máxima Verossimilhança consideraram cinco intervalos ad hoc. Como já ressaltado por Morillo (1999), entre outros, esta discretização não constitui um procedimento ideal já que, embora estes resultados sejam robustos frente à não linearidade, o procedimento de criação ad hoc de intervalos pode introduzir importantes vieses na estimação.

Por conta disso este estudo optou pela modelagem quantílica não linear conhecida como constrained B-spline smoothing (COBS). Esse instrumental é derivado do spline quantilico proposto por Koenker, NG e Portnoy (1994). Com ele, insere-se no âmbito da estatística não paramétrica. Em resumo, busca-se otimizar a função: 


$$
\min _{g \in \zeta} \sum_{i=1}^{N} \rho_{\tau}\left(y_{i}-g\left(x_{i}\right)\right)^{2}-\lambda \int\left|g^{\prime \prime}(x)\right| d x
$$

onde $\zeta$ corresponde a um Sobolev Space para funções diferenciáveis até a segunda ordem, g é uma função não paramétrica, $\lambda$ é um parâmetro de alisamento e $g$ "é a segunda derivada de g em relação a $x$. A solução para (11) é fornecida por He e $\mathrm{Ng}$ (1999), por meio de um processo de otimização linear.

Os resultados não paramétricos quantílicos são apresentados nas Figuras 3 e 4, no Apêndice. Assim como na estimação linear, consideraram-se os quantis $0.05,0.10,0.25,0.50,0.75,0.90$ e 0.95 . Os splines indicam uma relação linear até o quantil 0.25. A partir do quantil 0.45 , os dados apontam uma relação não linear. Todavia, estes resultados são mais robustos do que os lineares? Uma forma de compará-los é fornecida por Fan, Zhang e Zhang. (2001) a partir do seguinte teste de razão de verossimilhança generalizada:

$$
G L R=\frac{N}{2} \frac{S Q R-S Q I R}{S Q R}
$$

onde $S Q R$ é a soma dos quadrados dos resíduos do modelo quantílico linear e SQIR é a soma dos quadrados dos resíduos do modelo quantílico não paramétrico. Sob a hipótese nula de igualdade entre os dois métodos, Fan e Yao (2003) calculam a distribuição assintótica para (12) a partir do bootstrap. O teste foi aplicado para os quantis apresentados até então. Os resultados, sumarizados na Tabela 3, consideraram um bootstrap com 1000 réplicas.

Tabela 3 - Teste de Razão de Verossimilhança Generalizada

\begin{tabular}{c|c|c|c|c|c|c|c|c}
\hline Quantis & 0.05 & 0.10 & 0.25 & 0.45 & 0.50 & 0.75 & 0.90 & 0.95 \\
\hline$p$-value & 0.000 & 0.000 & 0.000 & 0.000 & 0.000 & 0.000 & 0.000 & 0.000 \\
\hline
\end{tabular}

Fonte: Elaborada pelos autores.

Como o $p$-value representa a probabilidade de se cometer o erro tipo I, ou seja, rejeitar a hipótese nula quando ela é verdadeira, constata-se que o modelo não linear é superior ao linear em todos os quantis considerados. Este resultado é coerente com o obtido por Laurini (2007).

Conforme apresentado na análise preliminar, a elevação dos índices de polarização da renda per capita fornece indícios da formação de clubes de convergência. No entanto, informações mais precisas podem ser encontradas observando os splines quantílicos dispostos no Apêndice (Figuras 3 e 4). Sabe-se que o processo de divergência, característico dos clubes de con- 
vergência, ocorre quando as curvas quantílicas convergem para locus diferenciados. Nesse sentido, uma inclinação descendente representa uma relação negativa entre o logaritmo da renda per capita inicial e a taxa de crescimento. Analogamente, uma inclinação ascendente indica uma relação positiva. O processo de divergência se verifica, portanto, quando os dois momentos estão presentes no conjunto dos splines não paramétricos quantílicos. No caso em estudo, pode-se notar que as curvas relacionadas aos quantis inferiores tendem para um polo "baixo", evidenciando uma menor taxa de crescimento para os municípios com maior logaritmo da renda per capita inicial. De forma oposta, os estratos superiores à mediana tendem para um polo "alto", caracterizando uma relação positiva entre tais variáveis. Sendo assim, percebe-se a existência de um processo divergente no que se refere à convergência de renda per capita entre os municípios nordestinos.

Essa análise nos fornece basicamente duas informações importantes. Primeiro, demonstra a insuficiência da modelagem quantílica linear já que, mesmo robusta frente à falácia de Galton, outliers e à heterocedasticidade, não consegue captar as não linearidades que põem em evidência os processos divergentes entre as curvas quantílicas, características da formação de clubes de convergência. Segundo, demonstra a formação de clubes de convergência entre os municípios nordestinos durante o período em estudo, assim como constatado em Laurini (2007) a partir dos dados brasileiros.

\section{Considerações Finais}

Este artigo desenvolve uma série de comparações entre métodos de inferência referentes à hipótese de convergência de renda per capita entre os municípios nordestinos, no período de 1970 a 1996. Partiu-se de um fato estilizado da literatura, qual seja: o método de mínimos quadrados ordinários padece de problemas relacionados à falácia de Galton, à presença de outliers e de heterocedasticidade.

Neste sentido, adotou-se o método de regressões quantílicas. Os resultados obtidos a partir dessa inferência corroboraram a hipótese de convergência plena entre os municípios. Contudo, como destacado em Laurini (2007), o processo de estimação via regressão quantílica linear assume a existência de relações gaussianas, de forma que o uso de tal método torna-se problemático caso esta forma funcional não seja verdadeira.

Sob estas constatações, tornou-se prudente a adoção de um instrumental não paramétrico. A partir de uma modelagem quantílica não linear, conhecida como constrained $B$-spline smoothing (COBS), observou-se que a taxa de crescimento do produto per capita e a renda inicial não possuem relação linear em todos os quantis examinados. O teste de razão de veros- 
similhança generalizada evidenciou que, para os dados em análise, as regressões quantílicas não paramétricas são mais robustas. Em resumo, a aplicação de tal método permitiu detectar a formação de clubes de convergência entre os municípios nordestinos.

\section{Referências}

ANDRADE, E. et al. Convergence clubs among Brazilian municipalities. Economics Letters, n.83, p. 179-184, 2004.

. Testing convergence across municipalities in Brazil using quantile regression. $\overline{\text { IBMEC }}$ Working Paper, n. 14, 2002.

BARRO, R. Economic growth in a cross-section of countries. The Quarterly Journal of Economics, n.106, p. 407-443, 1991.

DUCLOS, J. Y.; ESTEBAN, J.; RAY, D. Polarization: concepts, measurement, estimation. Econometrica, n.72, p. 1737-1772, 2004.

FAN, J.; ZHANG, C.; ZHANG, J. Generalized likelihood ratio statistics and Wilks phenomenon. The Annals of Statistics, n.29, p. 153-193, 2001.

FAN, J.; YAO, Q. Nonlinear time series: nonparametric and parametric methods. Springer, 2003.

FRIEDMAN, M. Do old fallacies ever die? Journal of Economic Literature, v. 30, n.4, p. 21292132, 1992.

HÄRDLE, W. Applied nonparametric regression. Cambridge: Cambridge University Press, 1990.

HE, X.; NG, P. COBS: qualitatively constrained smoothing via linear programming. Computational Statistics, n.14, p. 315-337, 1999.

KOENKER, R. Galton, Edgeworth, Frisch, and prospects for quantile regression in econometrics. Journal of Econometrics, n.95, p. 347-374, 2000.

KOENKER, R. Quantile regression. Cambridge: Cambridge University Press, 2005.

KOENKER, R.; BASSETT, G. Regression quantiles. Econometrica, n.46, p. 33-50, 1978.

. Robust tests for heteroscedasticity based on regression quantiles. Econometrica, n.50, p. 43-61, 1982.

KOENKER, R.; NG, P.; PORTNOY, S. Quantile smoothing splines. Biometrika, n.81, p. 673680, 1994.

LAURINI, M. A note on the use of quantile regression in beta convergence analysis. Economics Bulletin, n.3, p. 1-8, 2007.

LAURINI, M.; ANDRADE, E.; PEREIRA, P. Income convergence clubs for Brazilian municipalities: a non-parametric analysis. Applied Economics, n.37, p. 2099-2118, 2005.

MELO, M.; NOVO, A. The new empirics of economic growth: quantile regression estimation of growth equations. Unpublished working paper, 2002.

MELO, M.; PERELLI, R. Growth equations: a quantile regression exploration. The Quarterly Review of Economics and Finance, v. 43, n.4, p. 643-667, 2003. 
MORILO, D. Income mobility with nonparametric quantiles: a comparison of the U.S. and Germany. Working Paper: University of Illinois, 1999.

PORTO JÚNIOR, S.; RIBEIRO, E. Dinâmica espacial da renda per capita e crescimento entre os municípios da região nordeste do Brasil: uma análise markoviana. Revista Econômica do Nordeste, Fortaleza, v. 34, n.3, p. 405-420, 2003.

QUAH, D. Galton's fallacy and tests of convergence hypothesis. Scandinavian Journal of Economics, n.95, p. 427-443, 1993.

QUAH, D. Empirics for growth and distribution: stratification, polarization and convergence clubs. Journal of Economic Growth, n.2, p. 27-59, 1997.

SALA-I-MARTIN, X. The classical approach to convergence analysis. The Economic Journal, n.106, p. 1019-1036, 1996.

SOLOW, R. A contribution to the theory of economic growth. The Quarterly Journal of Economics, n.70, p. 65-94, 1956.

ZIEGELMANN, F. Nonparametric and semi-parametric methods in time series: the kernel smoothing approach. São Pedro: 10ª ESTE, 2003. 


\section{Apêndice}

\begin{tabular}{|c|c|c|c|c|c|c|c|c|c|c|}
\hline 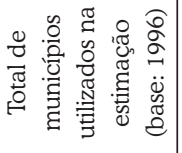 & $\stackrel{-}{ }$ & \& & $\stackrel{\infty}{\sim}$ & $\underset{\sim}{\vec{N}}$ & $\mathbb{N}$ & $\underset{\sim}{+\infty}$ & $\stackrel{\curvearrowright}{\vec{v}}$ & $\stackrel{n}{0}$ & $\stackrel{\sim n}{x}$ & \begin{tabular}{l}
$\infty$ \\
$\stackrel{2}{5}$ \\
\multirow{2}{*}{}
\end{tabular} \\
\hline 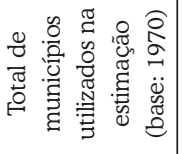 & $\Xi$ & $\stackrel{m}{m}$ & $\stackrel{\stackrel{P}{二}}{ }$ & 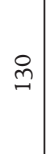 & $\stackrel{2}{-}$ & ț & $\stackrel{\cong}{\Xi}$ & $\stackrel{\circ}{\sim}$ & $\underset{N}{ }$ & $\begin{array}{l}\infty \\
\stackrel{0}{0} \\
\ddot{n}\end{array}$ \\
\hline 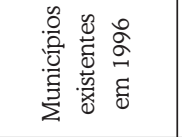 & $\underset{\sigma}{\sigma}$ & $\begin{array}{l}\stackrel{\sim}{\forall} \\
\vec{\nabla}\end{array}$ & $\underset{\sim}{\stackrel{D}{-}}$ & $\stackrel{n}{\sim}$ & $\tilde{N}$ & 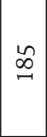 & $\overrightarrow{\mathrm{N}}$ & $\stackrel{\circ}{\circ}$ & $\stackrel{n}{n}$ & $\stackrel{0}{\stackrel{0}{N}}$ \\
\hline 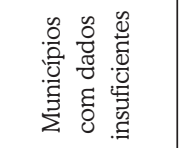 & 0 & $N$ & $\rightarrow$ & 0 & - & $\neg$ & $N$ & 0 & 0 & $n$ \\
\hline 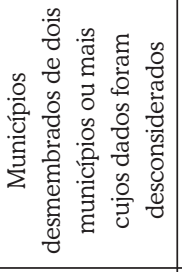 & $N$ & $\wedge$ & 0 & $\rightarrow$ & 0 & 0 & 0 & $\neg$ & 0 & 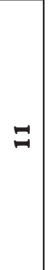 \\
\hline 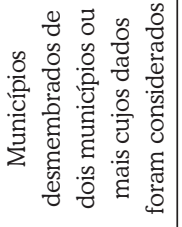 & $\rightarrow$ & 0 & 0 & $\stackrel{m}{\sim}$ & $\mathrm{N}$ & 0 & $\stackrel{\infty}{\sim}$ & 0 & 0 & $\exists$ \\
\hline 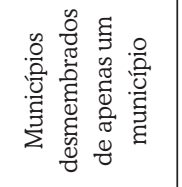 & in & $\approx$ & $\mathscr{f}$ & $\vec{\lambda}$ & in & 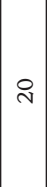 & $\stackrel{2}{1}$ & $\stackrel{\sim}{=}$ & - & $\stackrel{n}{n}$ \\
\hline 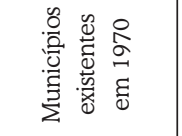 & む & $\stackrel{m}{m}$ & $\vec{J}$ & 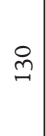 & $\vec{I}$ & $\begin{array}{l}n \\
0 \\
-1\end{array}$ & $\underset{\Xi}{\Xi}$ & $\stackrel{\circ}{\curvearrowleft}$ & $\underset{\sim}{ }$ & $\stackrel{n}{n}$ \\
\hline 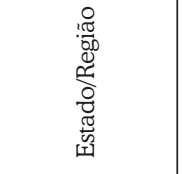 & 安 & 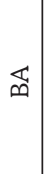 & 둰 & $\sum_{\Sigma}^{\mathbb{S}}$ & $\cong$ & 国 & $\Xi$ & Z & 떠ऽ & 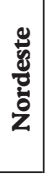 \\
\hline
\end{tabular}



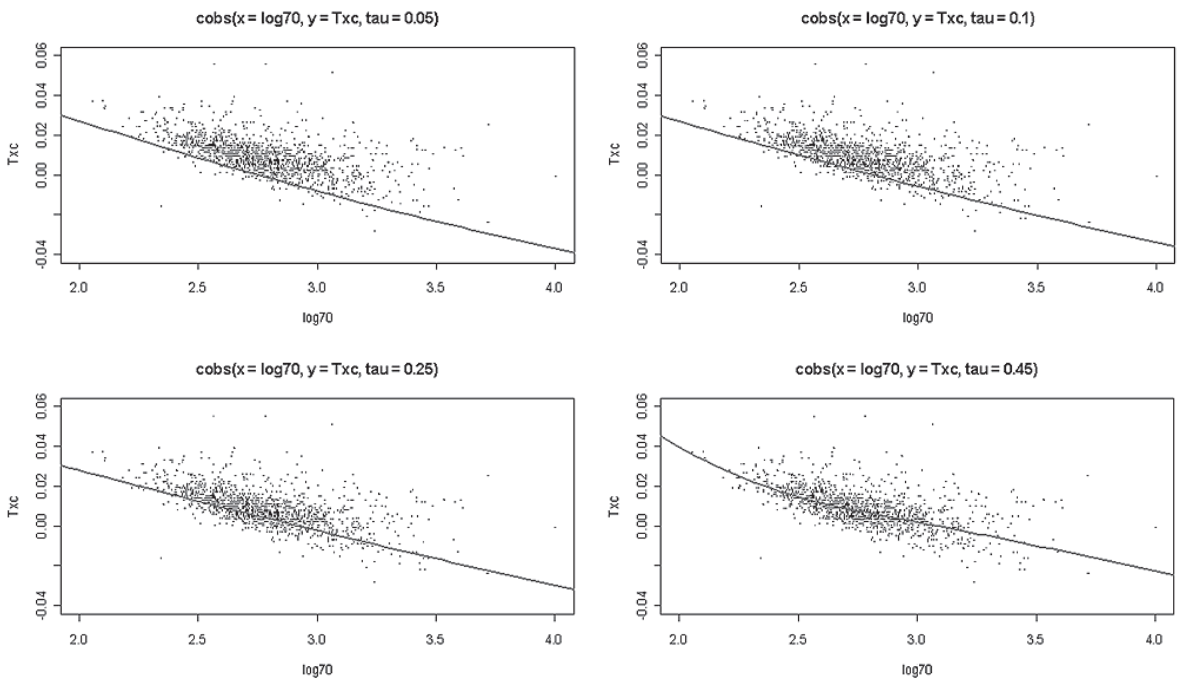

Figura 3 - Splines Quantílicos $(\tau=0.05,0.10,0.25,0.45)$

Fonte: Elaborada pelos autores.
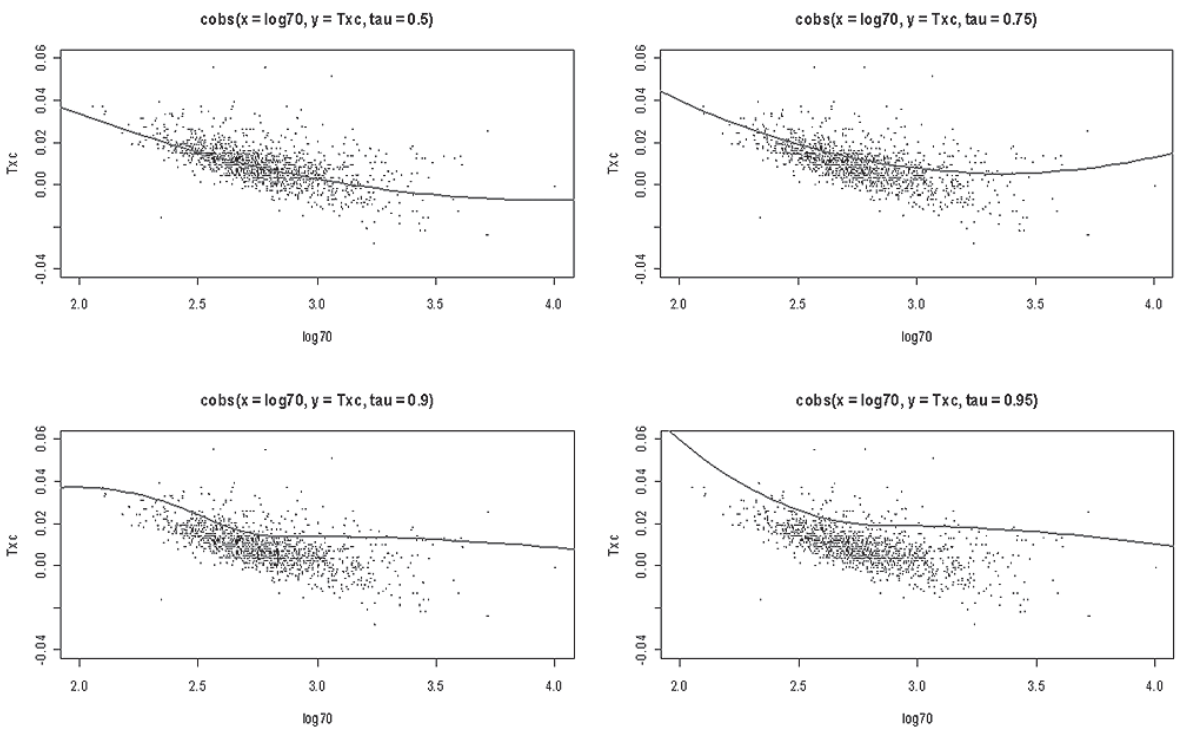

Figura 4 - Splines Quantílicos $(\tau=0.50,0.75,0.90,0.95)$

Fonte: Elaborada pelos autores.

Recebido em: 14/10/2008. Aceito em: 26/11/2008. 